\title{
Exposure to Leishmania spp. and sand flies in domestic animals in northwestern Ethiopia
}

Iva Rohousova ${ }^{1 *}$ (D), Dalit Talmi-Frank ${ }^{2 \dagger}$, Tatiana Kostalova $^{1 \dagger}$, Nikola Polanska $^{1}$, Tereza Lestinova ${ }^{1}$, Aysheshm Kassahun ${ }^{1}$, Daniel Yasur-Landau², Carla Maia ${ }^{1,3}$, Roni King ${ }^{4}$, Jan Votypka', Charles L. Jaffe ${ }^{5}$, Alon Warburg ${ }^{5}$, Asrat Hailu ${ }^{6}$, Petr Volf ${ }^{1}$ and Gad Baneth ${ }^{2^{*}}$

\begin{abstract}
Background: Human visceral leishmaniasis caused by Leishmania donovani is considered an anthroponosis; however, Leishmania-infected animals have been increasingly reported in L. donovani foci, and the role of these animals as reservoirs for human $L$. donovani infection remains unclear.

Methods: We conducted a study of domestic animals (goats, sheep, cows, dogs, and donkeys) in three L. donovani foci in northwestern Ethiopia. Domestic animals were screened for Leishmania DNA and for anti-L. donovani lgG. Serum anti-sand fly saliva antibodies were used as a marker of exposure to the vector sand fly, Phlebotomus orientalis.

Results: Of 546 animals tested, 32 (5.9\%) were positive for Leishmania DNA, with positive animals identified among all species studied. Sequencing indicated that the animals were infected with parasites of the L. donovani complex but could not distinguish between L. infantum and L. donovani. A total of $18.9 \%$ of the animals were seropositive for anti-L. donovani lgG, and $23.1 \%$ of the animals were seropositive for anti-P. orientalis saliva lgG, with the highest seroprevalence observed in dogs and sheep. A positive correlation was found between anti-P. orientalis saliva and anti-L. donovani lgGs in cows, goats, and sheep.
\end{abstract}

Conclusions: The detection of L. donovani complex DNA in the blood of domestic animals, the reported seroprevalence to the L. donovani antigen, and the widespread exposure to sand fly saliva among domestic animals indicate that they are frequently exposed to Leishmania infection and are likely to participate in the epidemiology of Leishmania infection, either as potential blood sources for sand flies or possibly as parasite hosts.

Keywords: Visceral leishmaniasis, Ethiopia, Domestic animals, Serology, PCR, Phlebotomus orientalis, Leishmania donovani, Sand fly saliva

\section{Background}

Leishmaniasis, a protozoan disease that is transmitted by sand flies (Diptera: Phlebotominae) and caused by parasites of the genus Leishmania (Kinetoplastida: Trypanosomatidae), is a neglected tropical and subtropical disease endemic to 98 countries worldwide. In East Africa, life-threatening human visceral leishmaniasis

\footnotetext{
* Correspondence: kolarova2011@gmail.com; gad.baneth@mail.huji.ac.il ${ }^{\dagger}$ Equal contributors

${ }^{1}$ Department of Parasitology, Faculty of Science, Charles University in Prague, Vinicna 7, 12844 Prague 2, Czech Republic

${ }^{2}$ School of Veterinary Medicine, The Hebrew University of Jerusalem, P.O. Box 12, Rehovot 76100, Israel

Full list of author information is available at the end of the article
}

(VL) is caused by Leishmania donovani and primarily affects the poor due to the lack of preventive measures and reduced access to health care facilities [1].

The optimal strategy for controlling this disease depends on understanding the epidemiology of VL, including its local transmission cycles. Leishmaniasis caused by L. donovani is believed to be an anthroponosis. However, in Latin America and the Mediterranean Basin, the closely related species L. infantum causes a zoonosis for which canids are the main reservoirs [2]. Controlling zoonoses involving domestic or sylvatic transmission requires a more complex intervention than would be necessary if humans were the only hosts. Several

\section{Biomed Central}


Leishmania-infected animals have been previously reported in L. donovani foci, including wild and domestic animals [3-5]. However, the role of these animals as parasite hosts or, possibly, as reservoirs for human $L$. donovani VL remains unclear and requires further examination.

Our study focused on the detection of Leishmania infections in domestic animals in three VL foci in northwestern Ethiopia. Domestic animals were screened for Leishmania DNA and anti-L. donovani IgG in their peripheral blood to detect infection and exposure to Leishmania, respectively. Additionally, anti-sand fly saliva antibodies were used as a marker of exposure [6] to Phlebotomus orientalis, the suspected vector of $L$. donovani in northwestern Ethiopia [7, 8]. The findings from this study could be used to further study the involvement of domestic animals in the transmission cycle of VL.

\section{Methods}

Study sites and sample collection

Animal blood and serum samples were collected in Addis Zemen, Humera, and Sheraro, three localities in northwestern Ethiopia endemic to human VL. In the Humera district (Tigray region), several outbreaks of VL have been recorded since 1970. Addis Zemen (Amhara region) and Sheraro (Tigray region) are sustained VL foci characterized by a local transmission cycle supported by migrant agricultural laborers returning from Humera [1].

Animal surveys were conducted during two field studies. In October 2010, 266 samples were collected in Addis Zemen and Sheraro, and in November 2010, an additional 280 samples were obtained in Humera (Table 1). For DNA extraction, samples of whole blood (with anticoagulant) were transported to the Hebrew University of Jerusalem (Israel), where extraction was performed. For serological testing, serum samples treated with a $1 \%$ azide solution were transported to Charles University in Prague (the Czech Republic) and stored at $-70{ }^{\circ} \mathrm{C}$.

Table 1 Serum samples collected from October to November 2010 in Ethiopian VL foci

\begin{tabular}{lllll}
\hline & Addis Zemen & Sheraro & Humera & Total \\
\hline Cow & 62 & 26 & 16 & 104 \\
Dog & 19 & 7 & 8 & 34 \\
Donkey & 3 & 11 & 6 & 20 \\
Goat & 0 & 106 & 133 & 239 \\
Sheep & 27 & 5 & 117 & 149 \\
Total & 111 & 155 & 280 & 546 \\
\hline
\end{tabular}

\section{Ethical approval}

The study was approved by the Ethiopian National Research Ethics Review Committee (NRERC), under approval no. 3.10/3398/04. Consent was obtained from the owners of the domestic animals for the collection of blood samples by a veterinarian. International animal experimentation guidelines were followed.

\section{DNA extraction and PCR amplification}

DNA was extracted from whole blood using the guanidine thiocyanate technique [9]. DNA was tested for Leishmania spp. infection via kDNA real-time PCR as previously described [10, 11]. Samples that tested positive were further tested by Leishmania internal transcribed spacer 1 (ITS1) real-time PCR and highresolution melt analysis (ITS1-HRM PCR) [12]. Samples that tested positive by ITS1-HRM PCR were further assessed via conventional PCR to amplify a larger segment of ITS1 [13]. All samples were tested in duplicate, and the results were compared with positive controls: $L$. infantum (MCAN/IL/2002/Skoshi), L. tropica (MHOM/ IL/2005/ LRC-L1239), and L. major (MHOM/TM/1973/ $5 \mathrm{ASKH})$ promastigotes. The negative controls included blood samples obtained from five Israeli dogs that had tested negative for Leishmania by PCR. All positive PCR products were submitted for DNA sequencing to the Center for Genomic Technologies at the Hebrew University of Jerusalem. The derived DNA sequences were compared with sequences in GenBank using the NCBI BLAST program (www.ncbi.nlm.nih.gov/BLAST). The percentage of positive animals for each species was calculated based on positive kDNA PCR results followed by sequencing. Samples were considered positive for Leishmania only if their kDNA sequence demonstrated the closest BLAST match to Leishmania and was at least $80 \%$ identical. A species was considered to be identified only when its ITS1 sequence shared 99 to $100 \%$ identity with an existing GenBank sequence.

\section{Discrimination between Leishmania infantum and Leishmania donovani}

As ITS1-HRM PCR does not discriminate between $L$. infantum and $L$. donovani infections [12], samples that tested positive for the $L$. donovani complex were further evaluated using conventional PCR to determine the species. Two independent PCR assays were carried out to amplify fragments of the Leishmania cysteine protease B (CPB) gene [14, 15]. Furthermore, amplification of the heat shock protein 70 (HSP70) gene, followed by restriction fragment length polymorphism analysis was also attempted for species discrimination [16]. The same positive and negative controls used for ITS1-HRM PCR were employed. 
A phylogenetic analysis was carried out using Kalign (www.ebi.ac.uk/tools/msa/kalign/) and BioEdit softwares. Only well-defined ITS sequences that were unambiguously assigned to the species $L$. donovani or L. infantum were downloaded from the GenBank database and used in the analysis (Additional file 1). The final alignment included 286 characters and is available upon request. Phylogenetic analyses of the ITS datasets were performed with PhyML for maximum likelihood (ML); the best-fitting model $[\mathrm{GTR}+I+\Gamma]$ of sequence evolution was assessed using Modeltest 3.7 software and bootstrapped with 1000 replicates.

\section{Anti-Leishmania donovani IgG antibodies}

An ELISA was used to measure specific anti-L. donovani IgG. Wells (CovaLink NH, Nunc) were coated with $L$. donovani promastigotes (Ethiopian strain MHOM/ET/ $67 / \mathrm{HU} 3,10^{5}$ cells per well) in $20 \mathrm{mM}$ carbonatebicarbonate buffer ( $\mathrm{pH} 9.25$ ) overnight at $4{ }^{\circ} \mathrm{C}$ and incubated with $6 \%$ blocking solution for $60 \mathrm{~min}$ at $37{ }^{\circ} \mathrm{C}$. Serum samples were diluted in $2 \%$ blocking solution and incubated in duplicate for $60 \mathrm{~min}$ at $37{ }^{\circ} \mathrm{C}$. Thereafter, peroxidase-conjugated secondary antibodies were added, followed by $45 \mathrm{~min}$ of incubation at $37{ }^{\circ} \mathrm{C}$. For details on the blocking solutions, sample dilutions, and conjugates employed in these assays, see Additional file 2. Absorbance was measured using a Tecan Infinite M200 microplate reader (Schoeller) at $492 \mathrm{~nm}$.

Hyperimmune sera from laboratory-bred mice experimentally infected with $L$. donovani served as positive controls. Negative serum samples were obtained from healthy cattle $(n=33)$, horses (as controls for the donkeys; $n=9)$, goats $(n=21)$, and sheep $(n=32)$ from the Czech Republic, which is a sand fly- and Leishmania-free country. Canine-negative $(\mathrm{n}=15)$ and canine-positive $(\mathrm{n}=2)$ control sera were obtained during a previous study [17] from laboratory-bred beagles with no history of exposure to sand flies or Leishmania or from Leishmaniapositive dogs, respectively.

\section{Anti-sand fly saliva IgG antibodies}

To estimate the exposure of domestic animals to $P$. orientalis, anti-saliva IgG antibodies were measured via ELISA. The same protocol applied for anti-Leishmania donovani IgG was used, with the following modifications: wells were coated with a salivary gland homogenate (corresponding to 0.2 gland/well, prepared as previously described [18]), and serum samples were incubated in duplicate for $90 \mathrm{~min}$ at $37{ }^{\circ} \mathrm{C}$. Hyperimmune sera from laboratory-bred mice exposed solely to $P$. orientalis served as a positive control. The same negative controls employed for the anti-L. donovani ELISA were also used here.
To assess the possible cross-reactivity of $P$. orientalis salivary gland homogenate with IgG antibodies against the saliva of other sand fly species, sera from mice and dogs that were experimentally exposed to a single sand fly species were used. Canine sera positive for anti- $P$. perniciosus and anti-L. longipalpis IgG antibodies were available from previous experiments in laboratory-bred beagles exposed solely to $P$. perniciosus [17] and L. longipalpis [18], respectively, the two proven vectors of $L$. infantum. The ELISA protocol described in Additional file 2 was applied with one modification: the sera were diluted 1:500. For the murine sera, the applied ELISA protocol was modified as follows: low-fat, dry milk (BioRad) was used as a blocking solution and diluent for the serum samples (1:200), and goat anti mouse IgG:HRP (AbD SEROTEC, STAR120P) diluted 1:1000, was used as a secondary antibody. The serum samples were obtained from BALB/c mice subjected to more than ten repeated exposures solely to $P$. orientalis (Ethiopia), $P$. papatasi (Turkey), P. duboscqi (Senegal), P. arabicus (Israel), or Sergentomyia schwetzi (Ethiopia). The experiments were approved by the Committee on the Ethics of Animal Experiments of Charles University in Prague (Permit Number: 24773/2008-10001) and were performed under a Certificate of Competency (Registration Number: CZU 934/05), in accordance with an Examination Order approved by the Central Commission for Animal Welfare of the Czech Republic.

\section{Statistical analysis}

For seroprevalence, cut-off values were calculated by the addition of three standard deviations to the mean optical density (OD) of the control sera. The differences in antibody levels between localities were analyzed using the nonparametric Wilcoxon Rank-Sum Test for Differences in Medians. Spearman's rank correlation matrix was used to assess the correlation between the variables. Statistical analyses were performed using NCSS 6.0.21 software, and the p-value was set at 0.05 .

\section{Results}

\section{Prevalence of Leishmania infection}

The overall prevalence of Leishmania DNA detected via PCR was $5.9 \%(32 / 546)$ (Table 2, Additional file 3). None of the 546 tested domestic animals presented visible clinical signs associated with leishmaniasis. Of the 32 animals that tested positive by kDNA PCR, nine were also positive for ITS1 PCR (Table 2, Additional file 3). The majority of Leishmania-positive animals (30 out of 32) were found in Humera, with the highest prevalence observed in cows $(18.8 \%)$. At the other localities, only one donkey in Sheraro and one dog in Addis Zemen were found to be positive for Leishmania (Table 2, Additional file 3). 
Table 2 Leishmania PCR positivity in samples from Ethiopian animals

\begin{tabular}{|c|c|c|c|c|c|}
\hline \multirow[b]{2}{*}{ Species } & \multicolumn{4}{|c|}{ Leishmania kDNA positive/total animals sampled (\% positive) } & \multirow{2}{*}{$\begin{array}{l}\text { Leishmania ITS1 positive (\% positive) } \\
\text { Total }\end{array}$} \\
\hline & Addis Zemen & Sheraro & Humera & Total & \\
\hline Cow & $0 / 62$ & $0 / 26$ & $3 / 16(18.8 \%)$ & 3/104 (2.9\%) & $1(1 \%)$ \\
\hline Dog & $1 / 19(5.3 \%)$ & $0 / 7$ & $1 / 8(12.5 \%)$ & $2 / 34(5.9 \%)$ & $1(2.9 \%)$ \\
\hline Donkey & $0 / 3$ & $1 / 11(9.1 \%)$ & $1 / 6(16.7 \%)$ & $2 / 20(10.0 \%)$ & 0 \\
\hline Goat & $0 / 0$ & $0 / 106$ & 16/133 (12.0\%) & $16 / 239(6.7 \%)$ & $3(1.3 \%)$ \\
\hline Sheep & $0 / 27$ & $0 / 5$ & $9 / 117(7.7 \%)$ & $9 / 149(6.0 \%)$ & $4(2.7 \%)$ \\
\hline Total & $1 / 111(0.9 \%)$ & $1 / 155$ (0.6 \%) & $30 / 280(10.7 \%)$ & $32 / 546(5.9 \%)$ & $9(1.6 \%)$ \\
\hline
\end{tabular}

A total of nine ITS1 DNA sequences, 265 bp long and $99 \%$ identical to L. infantum/L. donovani sequences, were obtained via ITS1-HRM-PCR. None of the animal samples yielded positive PCR results when targeting the $\mathrm{CPB}$ and HSP70 genes. A DNA sequence was obtained for only a single longer ITS1 amplicon from one sheep originating in Humera. This sequence (314 bp, [GenBank:KJ010540]) shares $100 \%$ identity with sequences from both L. infantum and L. donovani with $100 \%$

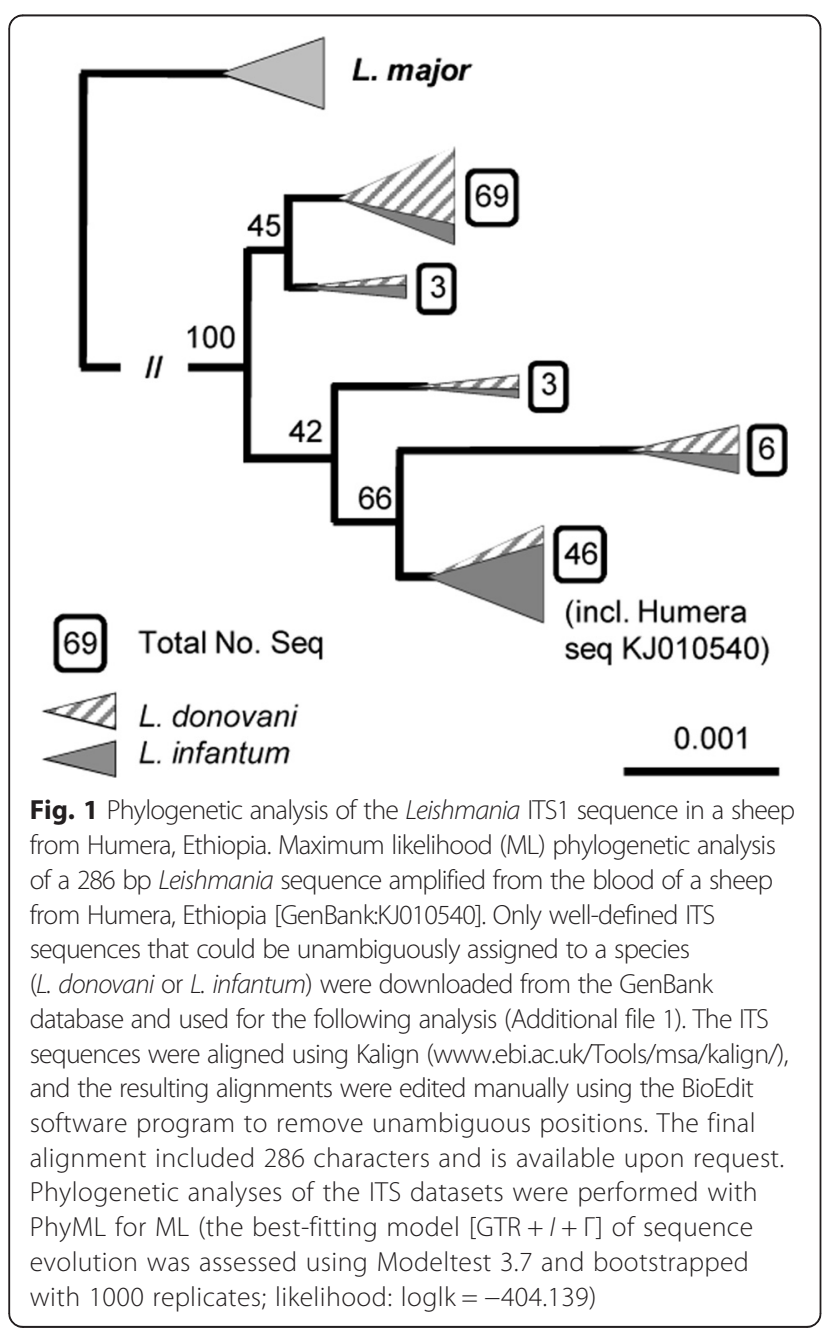

coverage, and its phylogeny did not permit discrimination between these two closely related species (Fig. 1).

\section{Anti-Leishmania donovani lgG antibodies}

Seropositive animals were found for every species tested. The overall seroprevalence of anti-L. donovani IgG in the Ethiopian samples was $18.9 \%(103 / 546)$ (Table 3; Fig. 2). Across all localities tested, the highest seropositivity was observed in dogs (overall $55.9 \%$ ) and the lowest in cows and donkeys (Table 3). Of the 32 animals that tested positive for Leishmania DNA, 12 animals also demonstrated seropositivity for the $L$. donovani antigen: 1 donkey, 3 goats, and 8 sheep (Additional file 3).

Apart from the cows, all of the Ethiopian animal species exhibited significantly higher levels of anti- $L$. donovani IgG compared with control animals (Fig. 2). Geographically, significantly higher levels of anti- $L$. donovani IgG were observed in all animal species from Humera and in dogs, goats, and sheep from the other localities tested, when compared with control animals (Fig. 2).

\section{Anti-Phlebotomus orientalis saliva IgG antibodies}

The seroprevalence of anti-P. orientalis IgG in Ethiopian animals was $23.1 \%(126 / 546)$ (Table 4). Seropositive animals were identified for every species and at every locality tested. In Addis Zemen and Sheraro, the highest seroprevalence was observed in dogs (57.9 and $57.1 \%$, respectively), whereas in Humera, the highest seroprevalence was among donkeys, dogs, and sheep (66.7, 62.5, and $57.3 \%$, respectively) (Table 4 ).

Apart from cows, all of the animal species from Ethiopia exhibited significantly $(\mathrm{p}<0.05)$ higher anti$P$. orientalis IgG seroreactivity compared with control animals (Fig. 2). Geographically, elevated levels of anti-P. orientalis IgG were observed in dogs, donkeys, and sheep from Humera and in dogs, donkeys, and goats from Sheraro. In Addis Zemen, only dogs exhibited significantly higher seroreactivity than control animals. The seroreactivities in the bovine 


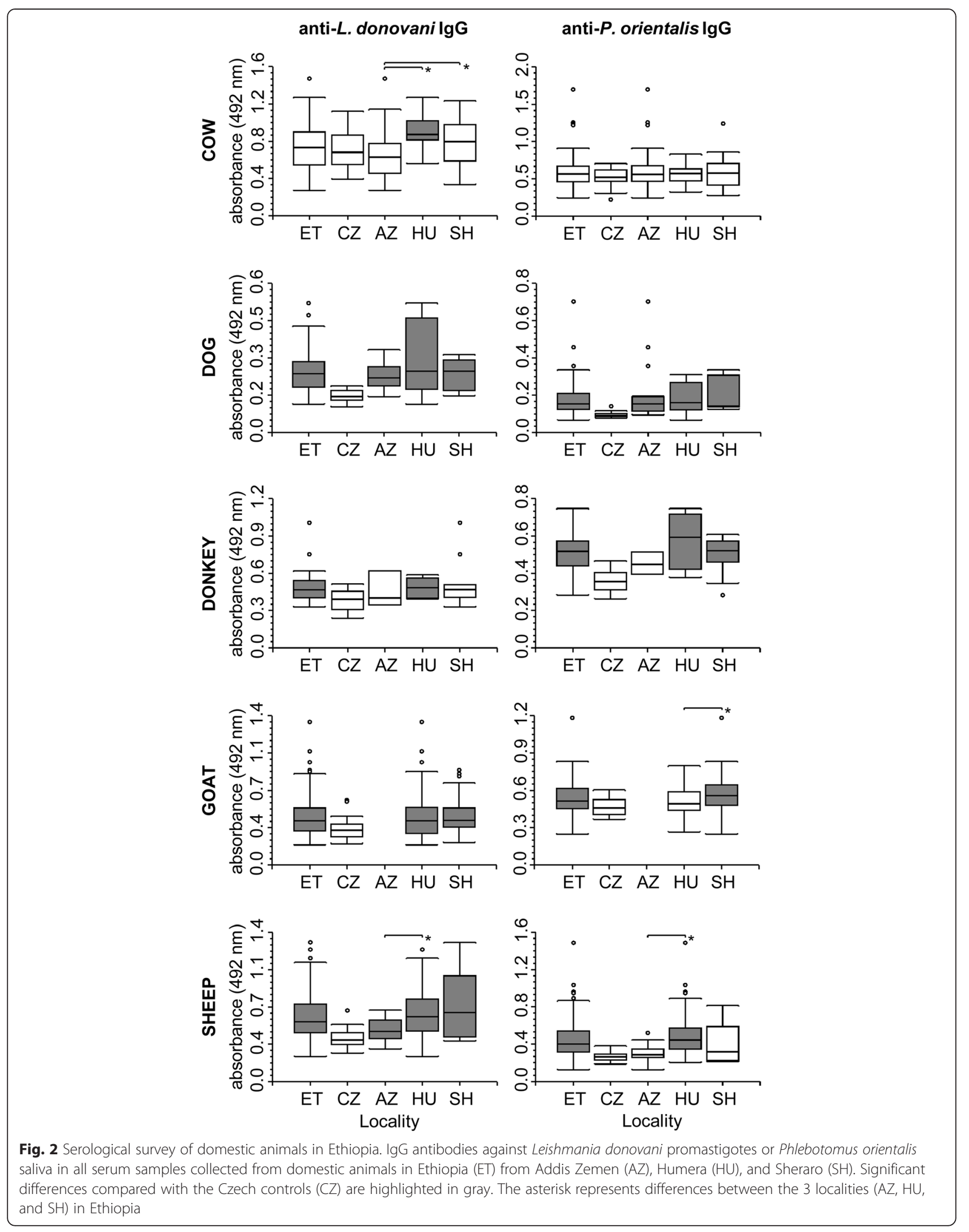


Table 3 Seropositivity of Ethiopian animals for Leishmania donovani lgG. The cut-off value was calculated as the mean optical density in the control animals plus 3 standard deviations (details provided in the Methods)

\begin{tabular}{llllll}
\hline & & \multicolumn{4}{l}{ Anti-L. donovani IgG positive/total animals sampled (\% seropositive) } \\
\cline { 3 - 6 } Species & Cut-off & Addis Zemen & Sheraro & Humera & Total \\
\hline Cow & 1.298 & $1 / 62(1.6 \%)$ & $0 / 26(0 \%)$ & $0 / 16(0 \%)$ & $1 / 104(1.0 \%)$ \\
Dog & 0.223 & $9 / 19(47.4 \%)$ & $5 / 7(71.4 \%)$ & $5 / 8(62.5 \%)$ & $19 / 34(55.9 \%)$ \\
Donkey & 0.652 & $0 / 3(0 \%)$ & $2 / 11(18.2 \%)$ & $0 / 6(0 \%)$ & $2 / 20(10.0 \%)$ \\
Goat & 0.675 & & $10 / 106(9.4 \%)$ & $15 / 133(11.3 \%)$ & $25 / 239(10.5 \%)$ \\
Sheep & 0.648 & $1 / 27(3.7 \%)$ & $3 / 5(60.0 \%)$ & $52 / 117(44.4 \%)$ & $56 / 149(37.6 \%)$ \\
Total & & $11 / 111(9.9 \%)$ & $20 / 155(12.9 \%)$ & $72 / 280(25.7 \%)$ & $103 / 546(18.9 \%)$ \\
\hline
\end{tabular}

samples were similar to those in control animals, regardless of the locality (Fig. 2).

To verify the specificity of the anti-P. orientalis saliva antibodies we used sera from dogs and mice that had been experimentally exposed to a single sand fly species. In dogs, the reactivity of anti-P. perniciosus and anti-Lutzomyia longipalpis sera against $P$. orientalis salivary gland homogenate (SGH) was similar to that for sera from non-exposed dogs (Fig. 3a). However, all of the selected canine sera of Ethiopian origin reacted strongly to $P$. orientalis SGH (Fig. 3a). In mice, the $P$. orientalis salivary antigen reacted strongly only to the homologous IgGs (Fig. 3b). The reactivities of all heterologous antigen-antibody combinations were similar to those for sera from nonexposed mice (Fig. 3b).

\section{Correlation analysis of serological results}

A positive correlation was found between the levels of anti- $P$. orientalis and anti-L. donovani IgG in Ethiopian cows $(\rho=0.37, p=0.0001)$, goats $(\rho=0.37, p<0.0001)$, and sheep $(\rho=0.65, \mathrm{p}<0.0001)$ (Table 5$)$. This correlation remained significant even when the locality was considered, except for the cows from Humera, for which the correlation was only slightly outside of the level of significance $(\rho=0.48, p=0.057)$. No significant correlation was found for the canine and donkey sera (Table 5).

\section{Discussion}

Visceral leishmaniasis is considered to be an anthroponosis in northwestern Ethiopia, but in nearby Sudanese foci, zoonotic transmission has also been suspected, with dogs and mongooses serving as possible reservoirs [3-5, 19]. With regard to domestic animals, sleeping near dogs, cattle, goats, or donkeys has been associated with an increased risk of VL in migrants and residents of Humera [20]. Understanding the mode of disease transmission, whether anthroponotic or zoonotic, is critical for the planning and implementation of effective VL control programs. Thus, one of the main goals of our study was to screen domestic animals for Leishmania DNA and discuss their possible involvement in the epidemiology of VL in Ethiopia as possible parasite hosts.

We evaluated two parameters associated with the ability of an animal to be a host for Leishmania parasites [21, 22]: (1) exposure to a sand fly vector as a source of blood and (2) the presence of Leishmania DNA in the animal's peripheral blood.

In northwestern Ethiopia, the sand fly vector species of $L$. donovani has not yet been identified. However, Phlebotomus orientalis is the most probable vector given that it has been found to be infected with $L$. donovani in nearby Sudanese foci [7] and its susceptibility to this Leishmania species has been demonstrated experimentally [8]. Exposure to P. orientalis was assessed using anti-sand fly saliva antibodies as a marker [6]. Anti-

Table 4 Seropositivity of Ethiopian animals for Phlebotomus orientalis saliva IgG. The cut-off value was calculated as the mean optical density in the control animals plus 3 standard deviations (details provided in the Methods)

\begin{tabular}{llllll}
\hline & & \multicolumn{4}{l}{ Anti-P. orientalis IgG positive/total animals sampled (\% seropositive) } \\
\cline { 3 - 6 } Species & Cut-off & Addis Zemen & Sheraro & Humera & Total \\
\hline Cow & 0.876 & $4 / 62(6.5 \%)$ & $1 / 26(3.8 \%)$ & $5 / 16(0 \%)$ & $5 / 104(4.8 \%)$ \\
Dog & 0.143 & $11 / 19(57.9 \%)$ & $4 / 7(57.1 \%)$ & $4 / 8(62.5 \%)$ & $20 / 34(58.8 \%)$ \\
Donkey & 0.550 & $0 / 3(0 \%)$ & $3 / 11(27.3 \%)$ & $166.7 \%)$ & $7 / 20(35.0 \%)$ \\
Goat & 0.685 & & $17 / 106(16.0 \%)$ & $6 / 133(4.5 \%)$ & $23 / 239(9.6 \%)$ \\
Sheep & 0.410 & $3 / 27(11.1 \%)$ & $1 / 5(20.0 \%)$ & $67 / 117(57.3 \%)$ & $71 / 149(47.7 \%)$ \\
Total & & $18 / 111(16.2 \%)$ & $26 / 155(16.8 \%)$ & $82 / 280(29.3 \%)$ & $126 / 546(23.1 \%)$ \\
\hline
\end{tabular}


saliva IgG antibodies were found in all of the animal species tested, which is indicative of the opportunistic feeding behavior of $P$. orientalis [23], thus meeting one criteria for the possible zoonotic transmission of $L$. donovani. Feeding preferences, together with other ecological constraints such as the localization of vector breeding sites [24] or vector susceptibility to harboring Leishmania infection [8] may help us to understand the complex picture of the ecology and transmission dynamics of VL in Ethiopia.

The presence of Leishmania DNA in animal peripheral blood and Leishmania seropositivity serve as reliable epidemiological markers for assessing infection. PCR positivity indicates the presence of the parasite $[25,26]$. Although this technique cannot prove the intact integrity of the parasite, viability of the detected Leishmania is highly probable given that its DNA degrades shortly after parasite death [27]. Seropositivity, on the other hand, is considered a marker of exposure to Leishmania infection [28]. The majority of Leishmania-positive animals were found in Humera, indicating dynamic transmission to domestic animals in this well-known active focus. However, many $L$. donovani-seropositive animals were found in all the three surveyed localities, suggesting that exposure to Leishmania parasites also occurred in the foci of Addis Zemen and Sheraro.

The fact that only one-third of the PCR-positive animals were positive for both kDNA and ITS1-HRM PCR, is not surprising because the ITS1 region has a considerably lower copy number $[11,12]$. Due to the small amount of parasite DNA available in blood samples, distinguishing between the closely related species $L$. donovani and $L$. infantum is notoriously difficult [15]. Moreover, distinction within the L. donovani complex in East Africa is controversial; strains that were previously split into $L$. donovani, $L$. archibaldi or $L$. infantum have now been classified into one group: L. donovani s.s. [29].

The most suspected animal reservoirs for L. donovani are dogs, which are known to play a key role as reservoir hosts in the transmission cycle of the closely related $L$. infantum [2, 30]. Several authors have reported PCRpositivity or seropositivity of dogs in L. donovani foci [3, 4, 19, 31-35], including Humera and Addis Zemen in Ethiopia [36-39]. In the present study, dogs demonstrated the highest Leishmania seroprevalence out of all the species tested at all study sites, with two PCRpositive dogs identified in Humera and Addis Zemen. As a suspected reservoir species, dogs are also highly attractive to the vector [35], which is supported by our findings that dogs exhibited the highest seroprevalence of anti- $P$. orientalis antibodies among the tested animal species. Most importantly, the same Leishmania strains have been recovered from dogs and VL patients $[3,4$,
19] and have been shown to persist in dogs for years [19]. Dogs have been recognized as a risk factor for human VL [20,37, 39], and as the most probable reservoir hosts, their involvement in disease transmission should be addressed in control strategies for VL caused by $L$. donovani.

Almost $38 \%$ of Leishmania-positive animals have also been found to be seropositive, indicating these domestic animals (donkeys, goats, sheep) as putative host species in local VL foci. Nevertheless, it is important to mention that neither PCR-positivity nor seropositivity indicates that an animal is able to maintain the parasite for a long period of time. This must be primarily demonstrated by the follow-up of infected animals. Several studies of naturally or experimentally infected non-canine domestic animals have demonstrated their different capabilities to maintain Leishmania infection. Cerqueira et al. [40] experimentally infected four donkeys with $L$. chagasi (syn. $L$. infantum). These donkeys remained seropositive until the end of the study, which lasted 12 months; however, the donkeys were able to overcome the infection and failed to infect the vector [40]. A PCR survey reported by Bhattarai et al. indicated that Leishmania infection in goats can persist for at least seven months [41]. On the other hand, $L$. donovani infection in sheep is likely time-limited because only one out of six experimentally infected sheep was shown to develop measurable amounts of anti-L. donovani antibodies and the transient presence of amastigotes in sampled tissue in a study that included 244 days of monitoring [42]. Thus, the $37.6 \%$ seropositivity detected in our study may indicate a high infection rate among Ethiopian sheep, further supported by the significantly higher levels of anti-L. donovani IgG antibodies among Leishmaniapositive sheep (Additional files 3 and 4).

The fact that many animals were seropositive for Leishmania while PCR-negative in the blood, and, on the other hand, that out of 32 PCR-positive animals, 20 animals were seronegative, could be explained by several possible mechanisms. Seropositivity and PCR-negativity might be attributable to infection in hosts that have resolved the infection but retain high titers of specific antibodies [40, 43]. Another possibility is that seropositive animals might carry the infection in their tissues without parasitemia and are therefore negative according to blood PCR [44]. The reverse situation with PCRpositivity and seronegativity could be attributable to the delayed development of a detectable antibody response in early infection [45], or due to an infection in animals whose B-cells are unresponsive to Leishmania antigens, as found in some asymptomatic hosts [2, 43, 44].

The role of other domestic animals as hosts or potential reservoirs for $L$. donovani is still unclear. The 

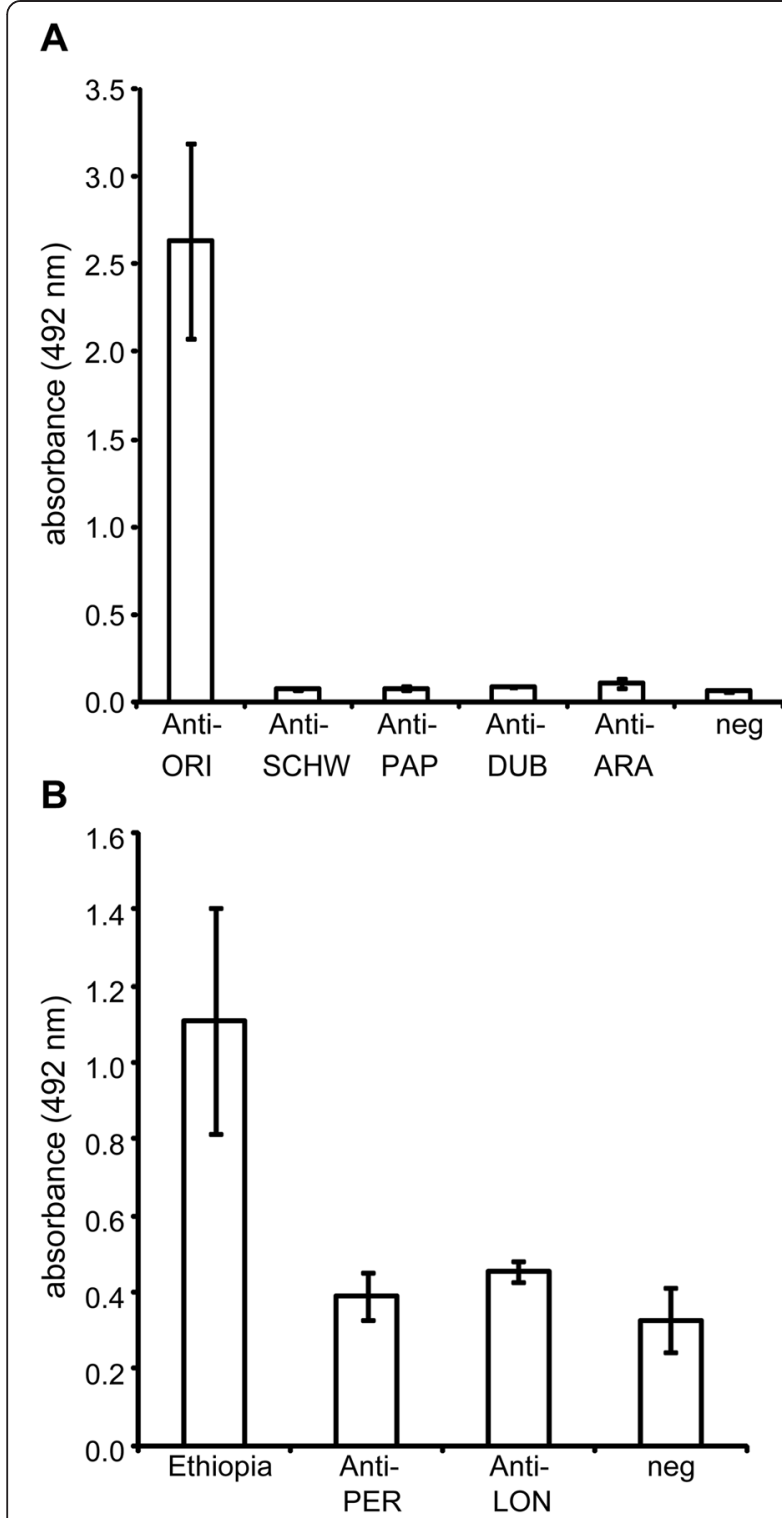

Fig. 3 Specificity of the anti-sand fly saliva lgG antibody response. The reactivity of Phlebotomus orientalis salivary gland homogenates $(\mathrm{SGH})$ with sera from mice $(\mathbf{a})$ and dogs $(\mathbf{b})$ repeatedly exposed to a single sand fly species was measured via ELISA. In graph A, SGH was incubated with sera from mice exposed to $P$. orientalis (Anti-ORI), Sergentomyia schwetzi (Anti-SCHW), P. papatasi (Anti-PAP), P. duboscai (Anti-DUB), or P. arabicus (Anti-ARA). Each bar represents the mean for two serum samples \pm the standard error. The values for the positive controls (the sera of mice incubated with homologous antigen) were as follows: $S$. schwetzi $=1.48 \pm 0.43$, P. papatasi $=$ $2.38 \pm 0.32$, P. duboscq $i=2.87 \pm 0.60$, and P. arabicus $=1.86 \pm 0.24$. In graph B, the SGH was incubated with the sera from dogs exposed to P. perniciosus (Anti-PER) or Lutzomyia longipalpis (Anti-LON). Seropositive Ethiopian dogs (Ethiopia) and dogs that had never been exposed to sand flies (neg) were used as positive and negative controls, respectively. Each bar represents the mean of five serum samples \pm the standard error. The absorbencies of the sera incubated with the homologous antigen were $2.42 \pm 0.06$ for L. longipalpis and $1.73 \pm 0.13$ for $P$. perniciosus
Table 5 Correlation analysis of serological results

\begin{tabular}{llllll}
\hline Species & & Ethiopia & Addis Zemen & Sheraro & Humera \\
\hline Cow & $\rho$ & $0.37^{* * *}$ & $0.38^{* *}$ & $0.43^{*}$ & 0.48 \\
& $\mathrm{n}$ & 104 & 62 & 26 & 16 \\
Dog & $\rho$ & 0.12 & 0.15 & -0.46 & 0.36 \\
& $\mathrm{n}$ & 34 & 19 & 7 & 8 \\
Donkey & $\rho$ & 0.31 & 0.50 & 0.52 & -0.03 \\
& $\mathrm{n}$ & 20 & 3 & 11 & 6 \\
Goat & $\rho$ & $0.37^{* * *}$ & & $0.36^{* * *}$ & $0.37^{* * *}$ \\
& $\mathrm{n}$ & 239 & & 106 & 133 \\
Sheep & $\rho$ & $0.65^{* * *}$ & $0.67^{* * *}$ & $1.00^{* * *}$ & $0.61^{* * *}$ \\
& $\mathrm{n}$ & 149 & 27 & 5 & 117 \\
\hline
\end{tabular}

Results from the Spearman-Rank Correlation Matrix test for anti-Leishmania donovani IgG and anti-Phlebotomus orientalis saliva IgG

$\rho$ correlation coefficient, $n$ number of serum samples tested

Asterisk $\left({ }^{*}\right)$ indicate significant correlations: ${ }^{*} p<0.05,{ }^{* *} p<0.01,{ }^{* * *} p<0.001$

present study is the first to report PCR-positive cattle, donkeys, goats, and sheep in Ethiopia. These animals, especially cattle, serve as sources of blood for $L$. donovani vectors [23, 46]. Even if these species do not serve as reservoir hosts for the parasite, they still attract large numbers of blood-questing female sand flies and may, therefore, act as a protective barrier in the case of resistant or refractory mammal species or as a risk factor in the case of susceptible species [30, 37, 47]. Prediction of the role of domestic animals in the amplification or dilution of VL risk might be possible using a recently described mathematical model for multi-host infectious diseases by applying relevant data [48].

In addition to the maintenance of persistent infection, the transmissibility competence, e.g. infectivity for the sand fly vector, is an important prerequisite for any mammal to serve as a Leishmania reservoir [28, 49]. These two criteria, among other aspects, can distinguish between a reservoir host and an incidental host that is not capable of infecting the vector [25]. Validation of these prerequisites for domestic animals in northwest Ethiopia, however, requires further investigation.

\section{Conclusions}

In conclusion, leishmaniasis caused by $L$. donovani is traditionally considered to be an anthroponosis in East Africa. However, the present study revealed widespread exposure to $L$. donovani and sand fly vector bites among domestic animals. The possible involvement of domestic animals as sources of blood for vector sand flies should therefore be considered in VL control strategies. However, the direct involvement of domestic animals in the transmission cycle of $L$. donovani warrants further 
investigation, most importantly by xenodiagnosis to determine their transmissibility competence.

\section{Additional files}

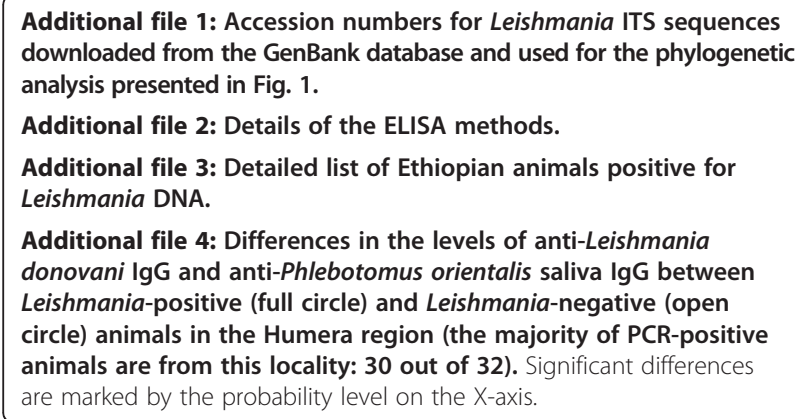

\section{Abbreviations}

ELISA: Enzyme-linked immunosorbent assay; ITS1: Internal transcribed spacer 1; kDNA: Kinetoplast deoxyribonucleic acid; L.: Leishmania or Lutzomyia; OD: Optical density; P.: Phlebotomus; PBS: Phosphate-buffered saline; PBS-Tw: Phosphate-buffered saline with Tween; PCR: Polymerase chain reaction; SGH: Salivary gland homogenate; VL: Visceral leishmaniasis.

\section{Competing interests}

The authors declare that they have no competing interests.

\section{Authors' contributions}

IR participated in the study design and field sample collection, carried out Leishmania serology, performed the statistical analysis, and drafted and finalized the manuscript. DTF and DYL carried out the Leishmania PCR and sequencing. TK, NP, and TL performed sand fly serology. AK, CM, RK, CLJ, and AW participated in field sample collection. JV carried out the sequence alignment and phylogenetic analysis. JV, AW, AH, and PV participated in the study design and coordination. GB conceived and designed the study, coordinated and participated in field work, and drafted and finalized the manuscript. DTF and TK contributed equally to the paper. All authors read and approved the final manuscript.

\section{Acknowledgements}

We thank our colleagues at the AAU-MF LRDL (Addis Ababa University Medical Faculty Leishmaniasis Research and Diagnostic Laboratory) as well as all of the drivers for their invaluable technical support during the field work. We are grateful to Vera Volfova for the maintenance of the $P$. orientalis colony. Animal control sera were generously provided by Dr. David Modry (Faculty of Veterinary Medicine, University of Veterinary and Pharmaceutical Sciences Brno, the Czech Republic) and Dr. Kamil Sedlak (State Veterinary Institute Prague, the (zech Republic).

This project was funded by the Bill and Melinda Gates Foundation, Global Health Program (OPPGH5336), the Czech Science Foundation (project no. 13-05292S), Charles University in Prague (GAUK 675012/B-BIO, SW260202), EurNegVec COST Action TD1303 and COST-CZ LD14076, and by EU grant FP7-261504 EDENext and is catalogued by the EDENext Steering Committee as EDENext273 (www.edenext.eu). The contents of this publication are the sole responsibility of the authors and do not necessarily reflect the views of the European Commission. The funders had no role in the study design, data collection and analysis, decision to publish, or preparation of manuscript. CM holds an FCT fellowship (SFRH/BPD/44082/2008). CLJ holds the Michael and Penny Feiwel Chair in Dermatology.

\section{Author details}

'Department of Parasitology, Faculty of Science, Charles University in Prague, Vinicna 7, 12844 Prague 2, Czech Republic. ${ }^{2}$ School of Veterinary Medicine, The Hebrew University of Jerusalem, P.O. Box 12, Rehovot 76100, Israel. ${ }^{3}$ Medical Parasitology Unit, Global Health and Tropical Medicine, Institute of Hygiene and Tropical Medicine, Universidade Nova de Lisboa, Rua da Junqueira 100, 1349-008 Lisboa, Portugal. ${ }^{4}$ Israel Nature and Parks Authority,
3 Am Ve'Olamo Street, Jerusalem 95463, Israel. ${ }^{5}$ Department of Microbiology and Molecular Genetics, The Institute for Medical Research Israel-Canada, The Kuvin Centre for the Study of Infectious and Tropical Diseases, The Hebrew University - Hadassah Medical School, The Hebrew University of Jerusalem, Jerusalem 91120, Israel. ${ }^{6}$ Department of Microbiology, Immunology and Parasitology, Faculty of Medicine, Addis Ababa University, P.O. Box 9086, Addis Ababa, Ethiopia.

Received: 11 May 2015 Accepted: 30 June 2015

Published online: 08 July 2015

\section{References}

1. Alvar J, Vélez ID, Bern C, Herrero M, Desjeux P, Cano J, et al. Leishmaniasis worldwide and global estimates of its incidence. PLoS One. 2012;7(5):e35671.

2. Baneth G, Koutinas AF, Solano-Gallego L, Bourdeau P, Ferrer L. Canine leishmaniosis - new concepts and insights on an expanding zoonosis: part one. Trends Parasitol. 2008;24(7):324-30.

3. Dereure J, Boni M, Pratlong F, El Hadi Osman M, Bucheton B, el-Safi S, et al. Visceral leishmaniasis in Sudan: first identifications of Leishmania from dogs. Trans R Soc Trop Med Hyg. 2000;94(2):154-5.

4. Baleela R, Llewellyn MS, Fitzpatrick S, Kuhls K, Schönian G, Miles MA, et al. Leishmania donovani populations in Eastern Sudan: temporal structuring and a link between human and canine transmission. Parasit Vectors. 2014;7(1):496.

5. Elnaiem DA, Hassan MM, Maingon R, Nureldin GH, Mekawi AM, Miles M et al. The Egyptian mongoose, Herpestes ichneumon, is a possible reservoir host of visceral leishmaniasis in eastern Sudan. Parasitology. 2001;122(Pt 5):531-6.

6. Rohousova I, Volf P. Sand fly saliva: effects on host immune response and Leishmania transmission. Folia Parasitologica. 2006;53(3):161-71.

7. Elnaiem DE. Ecology and control of the sand fly vectors of Leishmania donovani in East Africa, with special emphasis on Phlebotomus orientalis. J Vector Ecol. 2011;36 Suppl 1:S23-31.

8. Seblova V, Volfova V, Dvorak V, Pruzinova K, Votypka J, Kassahun A, et al. Phlebotomus orientalis sand flies from two geographically distant Ethiopian localities: biology, genetic analyses and susceptibility to Leishmania donovani. PLoS Negl Trop Dis. 2013:7(4):e2187.

9. Höss M, Pääbo S. DNA extraction from Pleistocene bones by a silica-based purification method. Nucleic Acids Res. 1993;21 (16):3913-4.

10. Nicolas L, Milon G, Prina E. Rapid differentiation of Old World Leishmania species by LightCycler polymerase chain reaction and melting curve analysis. J Microbiol Methods. 2002;51(3):295-9.

11. Talmi-Frank D, Jaffe CL, Nasereddin A, Warburg A, King R, Svobodova M, et al. Leishmania tropica in rock hyraxes (Procavia capensis) in a focus of human cutaneous leishmaniasis. Am J Trop Med Hyg. 2010;82(5):814-8.

12. Talmi-Frank D, Nasereddin A, Schnur LF, Schönian G, Töz SO, Jaffe CL, et al. Detection and identification of old world Leishmania by high resolution melt analysis. PLoS Negl Trop Dis. 2010:4(1):e581.

13. el Tai NO, Osman OF, el Fari M, Presber W, Schönian G. Genetic heterogeneity of ribosomal internal transcribed spacer in clinical samples of Leishmania donovani spotted on filter paper as revealed by single-strand conformation polymorphisms and sequencing. Trans R Soc Trop Med Hyg. 2000:94(5):575-9.

14. Hide M, Bañuls AL. Species-specific PCR assay for L. infantum/L. donovani discrimination. Acta Trop. 2006;100(3):241-5.

15. Zackay A, Nasereddin A, Takele Y, Tadesse D, Hailu W, Hurissa Z, et al. Polymorphism in the HASPB repeat region of East African Leishmania donovani strains. PLoS Negl Trop Dis. 2013;7(1):e2031.

16. Montalvo AM, Fraga J, Maes I, Dujardin JC, Van der Auwera G. Three new sensitive and specific heat-shock protein 70 PCRs for global Leishmania species identification. Eur J Clin Microbiol Infect Dis. 2012;31(7):1453-61.

17. Vlkova M, Rohousova I, Drahota J, Stanneck D, Kruedewagen EM, Mencke N, et al. Canine antibody response to Phlebotomus perniciosus bites negatively correlates with the risk of Leishmania infantum transmission. PLoS Negl Trop Dis. 2011;5(10):e1344

18. Hostomska J, Rohousova I, Volfova V, Stanneck D, Mencke N, Volf P. Kinetics of canine antibody response to saliva of the sand fly Lutzomyia longipalpis. Vector Borne Zoonotic Dis. 2008:8(4):443-50

19. Dereure J, El-Safi SH, Bucheton B, Boni M, Kheir MM, Davoust B, et al. Visceral leishmaniasis in eastern Sudan: parasite identification in humans and dogs; host-parasite relationships. Microbes Infect. 2003;5(12):1103-8. 
20. Argaw D, Mulugeta A, Herrero M, Nombela N, Teklu T, Tefera T, et al. Risk factors for visceral leishmaniasis among residents and migrants in Kafta-Humera, Ethiopia. PLoS Negl Trop Dis. 2013;7(11):e2543.

21. Chaves LF, Hernandez MJ, Dobson AP, Pascual M. Sources and sinks: revisiting the criteria for identifying reservoirs for American cutaneous leishmaniasis. Trends Parasitol. 2007:23(7):311-6.

22. Ashford RW. Leishmaniasis reservoirs and their significance in control. Clin Dermatol. 1996:14(5):523-32.

23. Gebre-Michael T, Balkew M, Berhe N, Hailu A, Mekonnen Y. Further studies on the phlebotomine sandflies of the kala-azar endemic lowlands of Humera-Metema (north-west Ethiopia) with observations on their natural blood meal sources. Parasit Vectors. 2010;3(1):6.

24. Moncaz A, Kirstein O, Gebresellassie A, Lemma W, Yared S, Gebre-Michael T, et al. Characterization of breeding sites of Phlebotomus orientalis - the vector of visceral leishmaniasis in northwestern Ethiopia. Acta Trop. 2014;139:5-14.

25. Silva ES, Gontijo CM, Melo MN. Contribution of molecular techniques to the epidemiology of neotropical Leishmania species. Trends Parasitol. 2005;21(12):550-2.

26. Oliveira FS, Brazil RP, Pacheco RS. Response to Silva et al.: Usefulness of PCR-based methods for screening Leishmania in epidemiological studies. Trends Parasitol. 2005;21(12):552-3.

27. Prina E, Roux E, Mattei D, Milon G. Leishmania DNA is rapidly degraded following parasite death: an analysis by microscopy and real-time PCR. Microbes Infect. 2007;9(11):1307-15.

28. Haydon DT, Cleaveland S, Taylor LH, Laurenson MK. Identifying reservoirs of infection: a conceptual and practical challenge. Emerg Infect Dis. 2002;8(12):1468-73.

29. Lukes J, Mauricio IL, Schönian G, Dujardin JC, Soteriadou K, Dedet JP, et al. Evolutionary and geographical history of the Leishmania donovani complex with a revision of current taxonomy. Proc Natl Acad Sci U S A. 2007;104(22):9375-80.

30. Bern C, Courtenay O, Alvar J. Of cattle, sand flies and men: a systematic review of risk factor analyses for South Asian visceral leishmaniasis and implications for elimination. PLoS Negl Trop Dis. 2010;4(2):e599.

31. Alam MZ, Yasin MG, Kato H, Sakurai T, Katakura K. PCR-based detection of Leishmania donovani DNA in a stray dog from a visceral leishmaniasis endemic focus in Bangladesh. J Vet Med Sci. 2013;75(1):75-8.

32. Rosypal AC, Tripp S, Kinlaw C, Hailemariam S, Tidwell RR, Lindsay DS, et al. Surveillance for antibodies to Leishmania spp. in dogs from Sri Lanka. J Parasitol. 2010;96(1):230-1.

33. Nawaratna SS, Weilgama DJ, Rajapaksha K. Cutaneous leishmaniasis in Sri Lanka: a study of possible animal reservoirs. Int J Infect Dis. 2009;13(4):513-7.

34. Sharma NL, Mahajan VK, Negi AK, Verma GK. The rK39 immunochromatic dipstick testing: a study for K39 seroprevalence in dogs and human leishmaniasis patients for possible animal reservoir of cutaneous and visceral leishmaniasis in endemic focus of Satluj river valley of Himachal Pradesh (India). Indian J Dermatol Venereol Leprol. 2009;75(1):52-5.

35. Hassan MM, Osman OF, El-Raba'a FM, Schallig HD, Elnaiem DE. Role of the domestic dog as a reservoir host of Leishmania donovani in eastern Sudan. Parasit Vectors. 2009;2(1):26.

36. Alvar J, Bashaye S, Argaw D, Cruz I, Aparicio P, Kassa A, et al. Kala-azar outbreak in Libo Kemkem, Ethiopia: epidemiologic and parasitologic assessment. Am J Trop Med Hyg. 2007;77(2):275-82.

37. Bashaye S, Nombela N, Argaw D, Mulugeta A, Herrero M, Nieto J, et al. Risk factors for visceral leishmaniasis in a new epidemic site in Amhara Region, Ethiopia. Am J Trop Med Hyg. 2009;81(1):34-9.

38. Kalayou S, Tadelle H, Bsrat A, Abebe N, Haileselassie M, Schallig HD. Serological evidence of Leishmania donovani infection in apparently healthy dogs using direct agglutination test (DAT) and rk39 dipstick tests in Kafta Humera, north-west Ethiopia. Transbound Emerg Dis. 2011;58(3):255-62.

39. Kenubih A, Dagnachew S, Almaw G, Abebe T, Takele Y, Hailu A, et al. Preliminary survey of domestic animal visceral leishmaniasis and risk factors in north-west Ethiopia. Trop Med Int Health. 2015;20(2):205-10.

40. Cerqueira EJ, Sherlock I, Gusmão A, Barbosa Júnior Ade A, Nakatani M. [Experimental infection of Equus asinus with Leishmania chagasi Cunha \& Chagas, 1937]. Rev Soc Bras Med Trop. 2003;36(6):695-701.

41. Bhattarai NR, Van der Auwera G, Rijal S, Picado A, Speybroeck N, Khanal B, et al. Domestic animals and epidemiology of visceral leishmaniasis, Nepal. Emerg Infect Dis. 2010;16(2):231-7.
42. Anjili CO, Ngichabe CK, Mbati PA, Lugalia RM, Wamwayi HM, Githure Jl. Experimental infection of domestic sheep with culture-derived Leishmania donovani promastigotes. Vet Parasitol. 1998;74(2-4):315-8.

43. Elmahallawy EK, Sampedro Martinez A, Rodriguez-Granger J, Hoyos-Mallecot Y, Agil A, Navarro Mari JM, et al. Diagnosis of leishmaniasis. J Infect Dev Ctries. 2014;8(8):961-72.

44. Miró G, Cardoso L, Pennisi MG, Oliva G, Baneth G. Canine leishmaniosis-new concepts and insights on an expanding zoonosis: part two. Trends Parasitol. 2008;24(8):371-7.

45. Simões-Mattos L, Mattos MR, Teixeira MJ, Oliveira-Lima JW, Bevilaqua CM, Prata-Júnior RC, et al. The susceptibility of domestic cats (Felis catus) to experimental infection with Leishmania braziliensis. Vet Parasitol. 2005;127(3-4):199-208.

46. Garlapati RB, Abbasi I, Warburg A, Poché D, Poché R. Identification of bloodmeals in wild caught blood fed Phlebotomus argentipes (Diptera: Psychodidae) using cytochrome b PCR and reverse line blotting in Bihar India. J Med Entomol. 2012;49(3):515-21.

47. Kolaczinski JH, Reithinger R, Worku DT, Ocheng A, Kasimiro J, Kabatereine N, et al. Risk factors of visceral leishmaniasis in East Africa: a case-control study in Pokot territory of Kenya and Uganda. Int J Epidemiol. 2008;37(2):344-52.

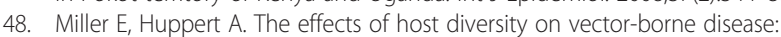
the conditions under which diversity will amplify or dilute the disease risk. PLoS One. 2013;8(11):e80279.

49. Roque $\mathrm{AL}$, Jansen $\mathrm{AM}$. Wild and synanthropic reservoirs of Leishmania species in the Americas. Int J Parasitol Parasites Wildl. 2014;3(3):251-62.

\section{Submit your next manuscript to BioMed Central and take full advantage of:}

- Convenient online submission

- Thorough peer review

- No space constraints or color figure charges

- Immediate publication on acceptance

- Inclusion in PubMed, CAS, Scopus and Google Scholar

- Research which is freely available for redistribution 